\title{
Capsule Commentary on Kimerling et al., Prevalence of Intimate Partner Violence among Women Veterans who Utilize Veterans Health Administration Primary Care
}

\author{
Holly N. Thomas, MD MS \\ Department of Medicine, University of Pittsburgh, Pittsburgh, PA, USA.
}

$\mathrm{T}$ he authors used a nationally representative sample $(N=6287)$ of women receiving primary care through the Veterans Health Administration (VHA) to (1) assess prevalence of intimate partner violence (IPV) in the past 12 months and (2) identify patient demographic, military, and health services utilization factors associated with pastyear IPV. Prevalence of past-year IPV was $18.5 \%$. Factors associated with IPV included economic hardship, sexual minority status, being a parent/guardian, past military sexual trauma, fewer years of military service, use of VHA as usual provider, higher health services utilization, and poorer continuity of care. ${ }^{1}$

The authors achieved a response rate of $84 \%$ and performed sensitivity analyses for non-response. They used a well-validated screening tool and included important covariates. Limitations include lack of data on veterans not using VHA and use of a telephone interview. Additionally, in studies estimating past-year rates, telescoping can result in inaccuracies. $^{2}$

In contrast to prior studies, rates of past-year IPV among female veterans were similar to the general population. This analysis may underestimate true prevalence. This survey only included women who had at least one primary care visit in the prior year; women experiencing IPV may be less likely to seek care. ${ }^{3}$ Additionally, women who are experiencing IPV may be more likely change contact information, making them more difficult to reach. ${ }^{4}$

This analysis highlights the significant relationship between socioeconomic hardship and IPV. Nearly $40 \%$ of homeless women reported past-year IPV. Financial stressors may make

Corresponding Author: Holly N. Thomas, MD MS; Department of MedicineUniversity of Pittsburgh, Pittsburgh, PA, USA (e-mail: thomashn@upmc.edu).

\section{Compliance with Ethical Standards:}

Conflicts of Interest: Dr. Thomas has no conflicts of interest to disclose.

\section{REFERENCES}

1. Kimerling R, Iverson KM, Dichter MF, Rodriguez AL, Wong A, Pavao J. Prevalence of intimate partner violence among women Veterans who utilize Veterans Health Administration primary care. J Gen Intern Med. doi:10. 1007/s11606-016-3701-7

2. Janssen SM, Chessa AG, Murre JM. Memory for time: how people date events. Mem Cognit. 2006;34(1):138-47.

3. Miller E, Decker MR, Raj A, Reed E, Marable D, Silverman JG. Intimate partner violence and health care-seeking patterns among female users of urban adolescent clinics. Matern Child Health J. 2010;14(6):910-7.

4. Nelson HD, Bougatsos C, Blazina I. Screening Women for Intimate Partner Violence and Elderly and Vulnerable Adults for Abuse: Systematic Review to Update the 2004 U.S. Preventive Services Task Force Recommendation. Rockville (MD); 2012.

5. McCauley J, Yurk RA, Jenckes MW, Ford DE. Inside "Pandora's box": abused women's experiences with clinicians and health services. J Gen Intern Med. 1998;13(8):549-55. 